\title{
Diabetes und Herzinsuffizienz
}

Philipp H. Baldia, Nikolaus Marx, Katharina A. Schütt

Der Diabetes mellitus ist eine wichtige Komorbidität bei Patienten mit Herzinsuffizienz. Liegen beide Erkrankungen gemeinsam vor, verschlechtert dies die Prognose der Patienten nachhaltig. Um das Outcome zu verbessern, sind eine suffiziente Diagnostik und eine differenzierte medikamentöse Therapie von entscheidender Bedeutung. Dieser Beitrag gibt einen Überblick über die Epidemiologie, Pathogenese, Diagnostik und therapeutischen Optionen.

\section{Einleitung}

Die Herzinsuffizienz als prognostisch wichtige Erkrankung für Morbidität und Mortalität bei Patienten mit Diabetes mellitus ist in den letzten Jahren zunehmend in den Fokus geraten. Die Tatsache, dass sich bei Patienten mit Diabetes mellitus eine Kardiomyopathie entwickeln kann, ohne dass eine koronare Herzerkrankung vorliegt, hat 1972 erstmalig zur Beschreibung der „diabetischen Kardiomyopathie“ geführt. Seitdem wird die Existenz dieses Krankheitsbildes höchst kontrovers diskutiert. Eine Ursache hierfür sind die immer noch nicht vollständig verstandenen, zugrunde liegenden pathophysiologischen Veränderungen. In der 2016 publizierten Leitlinie zur Herzinsuffizienz wurde neben den Krankheitsbildern Herzinsuffizienz mit erhaltener systolischer Funktion (HFpEF) und Herzinsuffizienz mit reduzierter systolischer Funktion (HFrEF) eine dritte Entität eingeführt: Herzinsuffizienz mit mittelgradig eingeschränkter systolischer Funktion (HFmrEF), die eine Ejektionsfraktion von 40$49 \%$ beschreibt [1]. Da alle bisher erhobenen Daten auf der alten Einteilung basieren, konzentriert sich der vorliegende Beitrag auf Patienten mit reduzierter bzw. erhaltener Pumpfunktion. Aufgrund der Relevanz des Diabetes mellitus für die Herzinsuffizienz sind die Therapiemöglichkeiten des Diabetes bei gleichzeitig bestehender Herzinsuffizienz ein weiteres Thema.

\section{Epidemiologie}

Der Diabetes mellitus ist ein weltweit zunehmendes Problem. Im Jahr 2015 waren weltweit über 415 Millionen Menschen im Alter über 20 Jahren betroffen und für das Jahr 2040 wird ein weiterer Anstieg auf 642 Millionen Individuen erwartet. Dabei entfielen 2017 allein auf Europa 66 Millionen Fälle, was einer Prävalenz von 9,1\% entspricht.

Die Prävalenz der Herzinsuffizienz hängt von der verwendeten Definition und der betrachteten Altersgruppe ab.
Insgesamt beträgt sie 1-2\% in der Gesamtbevölkerung; mit zunehmendem Alter nimmt sie auf über $10 \%$ bei den über 70-Jährigen zu. Bei Patienten mit Diabetes mellitus tritt die Herzinsuffizienz nicht nur häufiger, sondern vor allem bereits im jüngeren Lebensalter auf [2].

\section{Herzinsuffizienz bei Diabetes}

Verschiedene epidemiologische Studien der vergangenen Jahre belegen die Assoziation von Diabetes mellitus und einem erhöhten kardiovaskulären Risiko sowie einer häufigeren Herzinsuffizienz. Bereits eine gestörte Nüchternglukose, auch als „Prädiabetes“ bezeichnet, führt dazu, dass eine Herzinsuffizienz wahrscheinlicher wird (Hazard Ratio 1,2-1,7):

- Die multizentrische und multiethnische MESA-Studie (MESA = Multi-Ethnic Study of Atherosclerosis) schloss insgesamt 6814 Patienten mit KHK und einem Followup über 4 Jahre ein. Etwa 2 Drittel der Patienten entwickelten im Verlauf eine Herzinsuffizienz mit eingeschränkter linksventrikulärer Funktion, wobei das Risiko für Patienten mit Diabetes höher war als für Patienten mit Prädiabetes [3].

- In ähnlicher Weise untersuchte die Kaiser-PermanenteNorthwest-Datenbank an 17077 Probanden das Auftreten einer Herzinsuffizienz bei Individuen mit Diabetes mellitus im Vergleich zu alters- und geschlechtsgematchten Kontrollen. Die Studie demonstrierte eine 2,5-fach erhöhte Inzidenz der Herzinsuffizienz bei Diabetes mellitus: 30,9 je 1000 Patientenjahre bei Individuen mit Diabetes mellitus vs. 12,4 je 1000 Patientenjahre in der Kontrollgruppe [4].

- Ähnliche Zahlen ergaben sich in der Heart-and-SoulStudie, die Patienten mit stabiler KHK ohne Vorgeschichte einer Herzinsuffizienz über 4,1 Jahre untersuchte. Patienten mit Diabetes wiesen ein doppelt so hohes Risiko für die Entstehung einer Herzinsuffizienz auf als solche ohne Diabetes. Selbst nach Adjustierung für andere Risikofaktoren der Herzinsuffizienz wie Alter, Blutdruck oder Übergewicht verblieb der Diabetes als unabhängiger Risikofaktor (Hazard Ratio 3,34). 
Ferner zeigte sich eine Assoziation zwischen Erhöhung des HbA1c-Wertes und einer Hospitalisierung aufgrund von Herzinsuffizienz: Eine Erhöhung des $\mathrm{HbA}_{1 \mathrm{c}}$ um $1 \%$ ging mit einem um 36 \% erhöhten Risiko für eine Hospitalisierung einher [5].

- Die REACH-Registerstudie (REACH=The Reduction of Atherothrombosis for Continued Health) schloss insgesamt 45227 Patienten, davon 19699 mit Diabetes, ein und verfolgte diese über 4 Jahre nach. Auch hier konnte ein Diabetes mellitus als unabhängiger Risikofaktor für den kardiovaskulären Tod und Myokardinfarkt identifiziert werden. Ferner ergab sich ebenfalls eine um 33 \% höhere Hospitalisierungsrate gegenüber Individuen ohne Diabetes mellitus [6].

Diese Daten demonstrieren das deutlich erhöhte Risiko für Patienten mit Diabetes mellitus, eine Herzinsuffizienz zu entwickeln.

\section{Diabetes bei Herzinsuffizienz}

Betrachtet man umgekehrt die Prävalenz eines gestörten Glukosemetabolismus bzw. eines manifesten Diabetes mellitus in der Gruppe der Patienten mit Herzinsuffizienz, zeigt sich auch hier ein bedeutender Anteil von Patienten mit Prädiabetes und Diabetes:

- In einer prospektiven japanischen Studie untersuchten Matsue und Kollegen 2011 die Prävalenz eines bisher nicht diagnostizierten Prädiabetes bzw. Diabetes. Mehr als die Hälfte der Patienten (53,2\%) in der Studie wiesen entweder eine gestörte Glukosetoleranz oder einen manifesten Diabetes mellitus auf [7].

- Daten aus verschiedenen Registerstudien legen nahe, dass die Prävalenz des Diabetes bei Herzinsuffizienz zwischen 25 und $40 \%$ liegt [8].

- In ähnlicher Weise zeigte eine Analyse der PARADIGMHF-Studie (PARADIGM = Prospective Comparison of ARNI with ACEI to Determine Impact on Global Mortality and Morbidity in Heart Failure), dass $13 \%$ der Patienten einen bisher nicht diagnostizierten Diabetes mellitus aufwiesen und bei $25 \%$ ein Prädiabetes vorlag [9].

\section{Merke}

Bei Patienten mit Diabetes ist das Risiko einer Herzinsuffizienz bis zum 3-Fachen erhöht; umgekehrt haben Patienten mit Herzinsuffizienz häufig einen nicht diagnostizierten (Prä-) Diabetes.

\section{Prognose von Diabetes und Herzinsuffizienz}

Die Prognose von Patienten mit Herzinsuffizienz wird maßgeblich davon beeinflusst, ob ein Diabetes mellitus vorliegt:

- In der prospektiven dänischen DIAMOND-Studie (DIAMOND = Danish Investigations of Arrhythmia and Mortality on Dofetilide) wurden 5491 Patienten, die wegen einer kongestiven Herzinsuffizienz hospitali- siert worden waren, im Hinblick auf das mit Diabetes assoziierte Mortalitätsrisiko untersucht. Die 1-JahresMortalität lag bei den Patienten mit Diabetes mellitus bei $31 \%$; bereits 3 Jahre nach Einschluss waren mehr als $50 \%$ der Patienten mit Diabetes verstorben. Das relative Risiko zu versterben lag, selbst nach Adjustierung für ischämische Vorerkrankungen und vorausgegangene Infarkte, bei Patienten mit Diabetes $50 \%$ höher als bei Patienten ohne Diabetes [10].

- Einen ähnlich schwerwiegenden Einfluss des Diabetes auf die Mortalität bei herzinsuffizienten Patienten ergaben auch andere große Herzinsuffizienz-Studien wie SAVE (Survival and Ventricular Enlargement), CHARM (Candesartan in Heart failure Assessment of Reduction in Mortality and Morbidity) und VALIANT (Valsartan in Acute Myocardial Infarction). Das kumulative Risiko, innerhalb von 3 Jahren an einem kardiovaskulären Tod zu versterben oder aufgrund einer Herzinsuffizienz hospitalisiert zu werden, betrug in allen Studien $40 \%$. Dabei war das Risiko sowohl für Männer und Frauen mit Diabetes als auch für Patienten mit HFpEF und HFrEF erhöht.

- Zur genaueren Unterscheidung zwischen herzinsuffizienten Patienten mit erhaltener und solchen mit eingeschränkter Ejektionsfraktion analysierte die CHARM-Studie Unterschiede zwischen Individuen mit und ohne Diabetes. Patienten mit Diabetes und eingeschränkter Pumpfunktion hatten dabei die schlechteste Prognose im Hinblick auf die kardiovaskuläre Mortalität (119,1 Ereignisse je 1000 Patientenjahre vs. 75,7 Ereignisse je 1000 Patientenjahre). Eine ähnlich schlechte Prognose, verglichen mit Patienten ohne Diabetes, wiesen solche mit Diabetes und erhaltener Pumpfunktion auf (58,6 je 1000 Patientenjahre vs. 31,1 je 1000 Patientenjahre) ( $\triangleright$ Abb. 1). Das Hospitalisierungsrisiko von an Diabetes erkrankten Patienten war ebenfalls doppelt so hoch wie bei den nicht an Diabetes erkrankten Individuen. Insbesondere bei erhaltener Pumpfunktion lag das Risiko der Patienten mit Diabetes höher (154,4 je 1000 Patientenjahre vs. 84,3 je 1000 Patientenjahre) - sie wiesen insgesamt unabhängig von der Pumpfunktion ein doppelt so hohes Hospitalisierungsrisiko auf.

- Neuere Daten aus der PARADIGM-HF-Studie zeigen eine deutlich erhöhte kardiovaskuläre Mortalität bei Patienten mit Herzinsuffizienz und Diabetes im Vergleich zu solchen ohne Diabetes mit einer kardiovaskulären Sterblichkeit von $17 \%$ über 27 Monate [9].

Anhand dieser Studien lässt sich der wichtige Einfluss eines Diabetes auf die Prognose von herzinsuffizienten Patienten belegen: Innerhalb 1 Jahres kommt es bei 12-15\% der Patienten zu einer Hospitalisierung aufgrund ihrer Herzinsuffizienz oder zum kardiovaskulären Tod. 


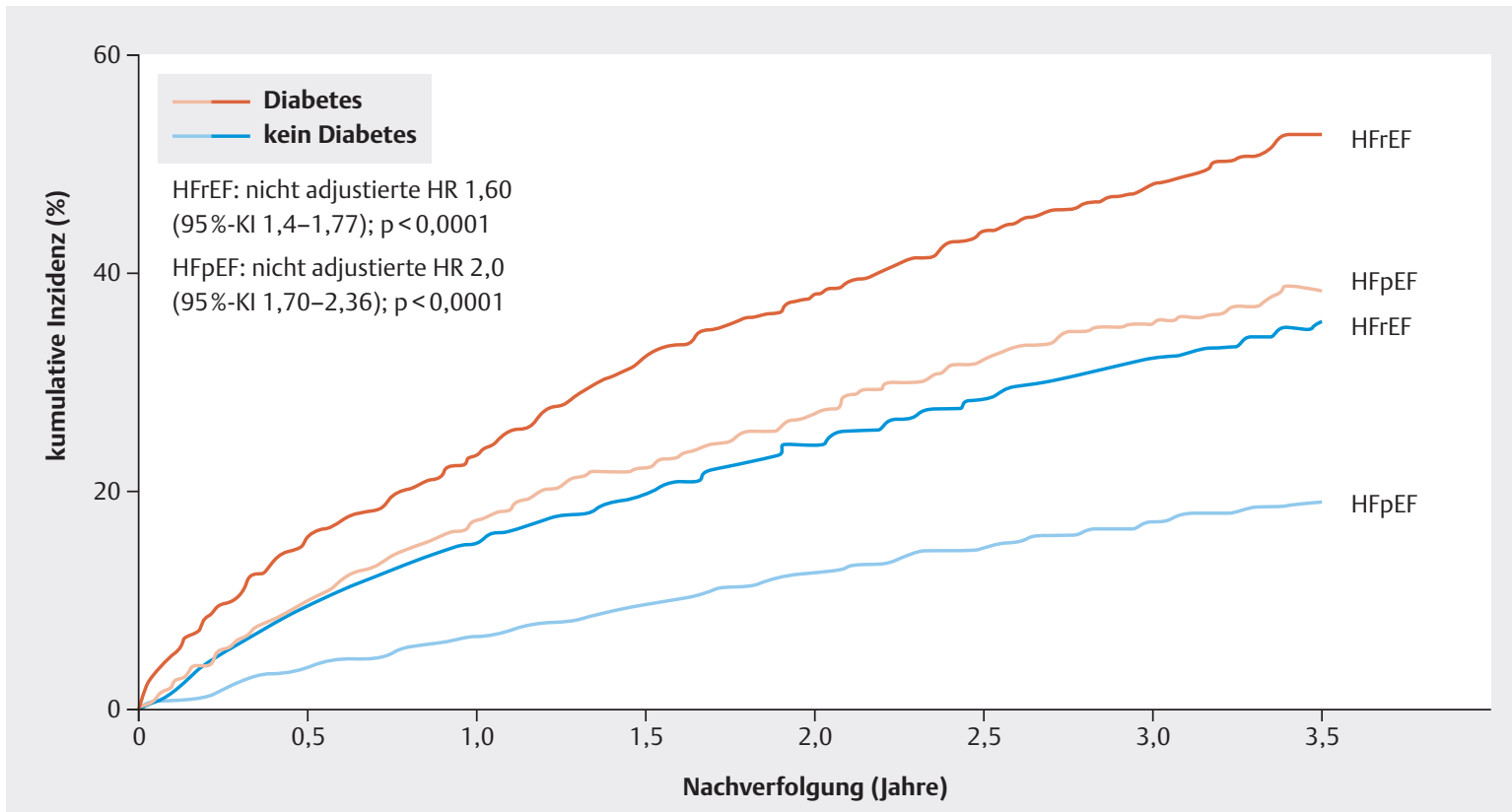

- Abb. 1 Kardiovaskulärer Tod oder Hospitalisierung aufgrund von Herzinsuffizienz bei Patienten mit und ohne Diabetes mellitus je nach Typ der Herzinsuffizienz (HFrEF oder HFpEF).

Merke

Allein das Vorliegen eines Diabetes ist unabhängig von der linksventrikulären Pumpfunktion mit einer Prognoseverschlechterung der Herzinsuffizienz verbunden.

\section{Pathophysiologie}

Neben den klassischen Risikofaktoren für das Entstehen einer Herzinsuffizienz tragen bei der diabetischen Kardiomyopathie viele, noch nicht vollständig verstandene Faktoren zur Pathophysiologie bei. Insbesondere stehen metabolische Veränderungen auf systemischer und lokaler myokardialer Ebene im Vordergrund. Dabei sind vor allem systemische Veränderungen des Metabolismus mit einer systolischen Dysfunktion des Myokards assoziiert, genauso wie lokale Störungen des myokardialen Metabolismus bzw. der myokardialen Homöostase mit einer diastolischen Dysfunktion. $>$ Abb. 2 gibt einen vereinfachten Überblick über einzelne pathogene Faktoren.

\section{Diagnostik}

\section{Diagnostik der Herzinsuffizienz}

\section{Allgemeines Vorgehen}

Bei der Diagnostik der Herzinsuffizienz wird keine Unterscheidung zwischen Patienten mit und ohne Diabetes getroffen [1].
Die Basis der Diagnostik bildet eine ausführliche Anamneseerhebung hinsichtlich klassischer Symptome: körperliche Belastbarkeit nach Einteilung in NYHA-Stadien (NYHA = New York Heart Association), Nykturie, Gewichtszunahme. Auch nach möglichen ätiologisch verantwortlichen Begleitfaktoren wird gefragt, z. B. nach einer KHK, einer arteriellen Hypertonie, einem Drogenabusus oder einer zurückliegenden medikamentösen Therapie mit kardiotoxischen Substanzen.

\section{PRAXISTIPP}

Gerade bei Patienten mit Diabetes ist die Diagnose einer Herzinsuffizienz oft schwierig zu stellen, weil die vom Patienten geäußerten Symptome unspezifisch sind und auch andere Erkrankungen Dyspnoe, Müdigkeit und Leistungsminderung verursachen können.

Die nächsten Schritte sind eine körperliche Untersuchung, bei der man insbesondere auf Kongestionszeichen achtet, und ein EKG, mit dem eine mögliche Linksherzhypertrophie, Erregungsrückbildungsstörungen als Zeichen einer Ischämie bzw. eines vorangegangenen Infarktes oder andere Gründe für eine Herzfunktionseinschränkung festgestellt werden können.

Erhärtet sich nach o. g. Untersuchungen der Verdacht einer Herzinsuffizienz, schließen sich eine laborchemische Analyse der natriuretischen Peptide (NT-proBNP oder 


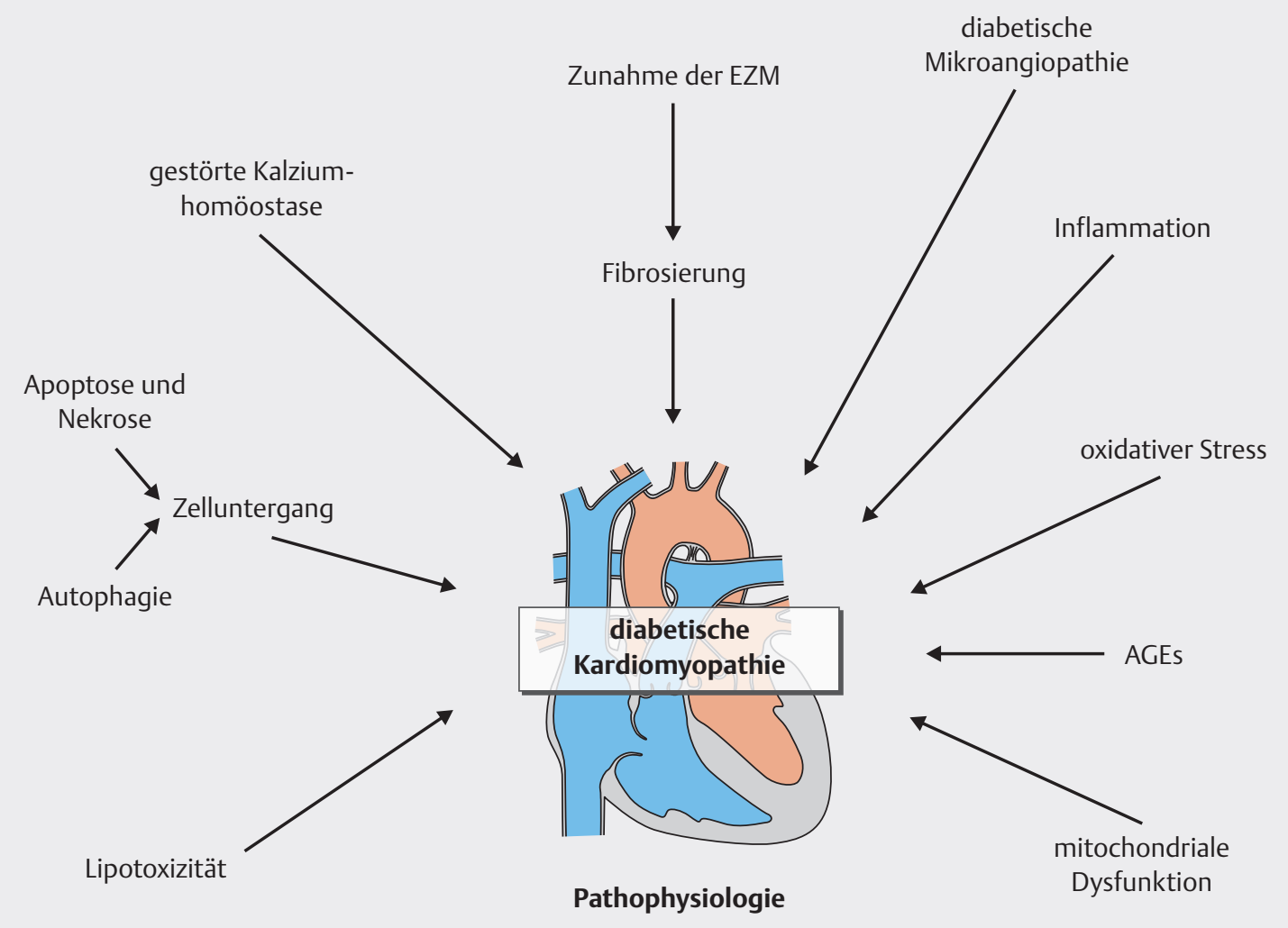

- Abb.2 Pathophysiologie der diabetischen Kardiomyopathie. AGE = „advanced glycated endproduct“; ROS = „reactive oxygen species“. Quelle Herz: Middeke M. Anatomie. In: FüeßI H, Middeke M, Hrsg. Duale Reihe Anamnese und Klinische Untersuchung. 6., aktualisierte Auflage. Stuttgart: Thieme 2018. doi:10.1055/b-006-149437.

BNP) und eine Echokardiografie an. Anhand der echokardiografisch quantifizierten linksventrikulären Pumpfunktion teilt man die Herzinsuffizienz in HFpEF (LVEF $\geq 50 \%$ ), HFmrEF (LVEF $\geq 40$ und $\leq 49 \%$ ) oder HFrEF (LVEF < $40 \%$ ) ein.

Im Anschluss an die Diagnosefindung ist ggf. eine weitere ätiologische Abklärung (z. B. mittels Links-/Rechtsherzkatheteruntersuchung, kardialer MRT oder Myokardbiopsie) sowie die Initiierung einer adäquaten Therapie indiziert.

\section{Diagnostik der Herzinsuffizienz}

mit erhaltener Pumpfunktion (HFpEF)

Während sich die Diagnose einer HFrEF mittels Echokardiografie verhältnismäßig einfach gestaltet, ist die Diagnose einer HFpEF bei erhaltener linksventrikulärer Pumpfunktion schwieriger zu stellen. Gemäß ESC wird empfohlen, folgende diagnostische Kriterien anzuwenden:

- Symptome der Herzinsuffizienz,

- erhaltene linksventrikuläre Pumpfunktion (LVEF $\geq 50 \%$ oder $40-49 \%$ bei HFmrEF),

- erhöhte Spiegel natriuretischer Peptide (BNP > 35 pg/ml und/oder NT-proBNP > 125 pg/ml),
- objektiver Nachweis struktureller oder funktioneller Veränderungen, z. B.:

- ein echokardiografisch erhöhter linksatrialer Volumenindex $(\mathrm{LAVI})>34 \mathrm{ml} / \mathrm{m}^{2}$ oder

- ein erhöhter linksventrikulärer Massenindex (LVMI) $\geq 115 \mathrm{~g} / \mathrm{m}^{2}$ bei Männern und $\geq 95 \mathrm{~g} / \mathrm{m}^{2}$ bei Frauen oder

- eine linksventrikuläre Hypertrophie bzw. Dilatation der Herzhöhlen sowie

- echokardiografische Zeichen der diastolischen Dysfunktion, z. B. E/é $\geq 13$ und ein mittleres é im Bereich des Septums und der Lateralwand $<9 \mathrm{~cm} / \mathrm{s}$.

Ein zeitgleich vorliegendes Vorhofflimmern erschwert die Diagnose der HFpEF. So kann es beim Vorhofflimmern zu einer Dilatation des linken Atriums und damit einhergehend des LAVIs kommen. Auch eine Erhöhung der natriuretischen Peptide ist möglich. In der Echokardiografie fällt die Quantifizierung der diastolischen Parameter im Rahmen des Vorhofflimmerns schwerer. Ferner kann ein Vorhofflimmern nicht nur eine Herzinsuffizienz verursachen (z. B. Tachykardiomyopathie), sondern auch selbst Zeichen einer zugrunde liegenden Herzinsuffizienz sein. 
Merke

Die Kombination aus klinischen Symptomen und bildgebenden Verfahren spielt eine Schlüsselrolle in der Diagnostik der Herzinsuffizienz mit erhaltener Pumpfunktion.

\section{Diagnostik des Diabetes}

Da die Prävalenz des Diabetes mellitus bei Patienten mit Herzinsuffizienz sehr hoch ist (25-40\% [8]), sollte bei Patienten mit diagnostizierter Herzinsuffizienz und bisher nicht bekanntem Diabetes mellitus ein DiabetesScreening erwogen werden. Entsprechend der neuesten DDG-Praxisempfehlung (DGG = Deutsche Diabetes Gesellschaft) kann ein Diabetes mellitus wie folgt diagnostiziert werden:

- Bei Symptomen eines Diabetes mellitus (z. B. Gewichtsverlust, Polydipsie, Polyurie), einem erhöhten Risiko für Diabetes (z. B. Diabetes-Risiko-Test) oder einem auffälligen Glukose- bzw. $\mathrm{HbA}_{1 c}$-Wert ist eine weitere Diagnostik mittels Nüchtern-Plasmaglukose oder Gelegenheitsplasmaglukose indiziert.

- Als sicher diagnostiziert gilt ein Diabetes, wenn entweder eine pathologische Nüchtern-Plasmaglukose oder Gelegenheitsplasmaglukose erhoben wird:

- Nüchtern-Plasmaglukose (NPG) $\geq 126 \mathrm{mg} / \mathrm{dl}$ (nüchtern bedeutet keine Kalorienaufnahme binnen der letzten 8 Stunden vor dem Test),

- Gelegenheitsplasmaglukose $\geq 200 \mathrm{mg} / \mathrm{dl}$.

- In Grenzfällen und bei weiterhin bestehendem klinischem Verdacht ist eine weitere Abklärung mittels oralem Glukosetoleranztest (oGTT) oder $\mathrm{HbA}_{1 \mathrm{c}}$-Wert angeraten. Hier gelten folgende Werte als sicherer Nachweis eines Diabetes mellitus:

- 2-Stunden-Plasmaglukose $\geq 200 \mathrm{mg} / \mathrm{dl}$ während eines oralen Glukosetoleranztests (OGTT) und - $\mathrm{HbA}_{1 \mathrm{c}} \geq 6,5 \%$.

Als Prädiabetes definiert man folgende Testergebnisse:

- NPG zwischen 100 und 125 mg/dl (bzw. 110-125 mg/dl gemäß WHO),

- 2-Stunden-Plasmaglukose 140-199 mg/dl und

- $\mathrm{HbA}_{1 \mathrm{c}} 5,7-6,4 \%$.

\section{PRAXISTIPP}

Der $\mathrm{HbA}_{1 \mathrm{c}}$-Wert ist nach aktuellen DDG-Empfehlungen nicht als alleiniger Nachweis eines Diabetes mellitus empfohlen, da es zurzeit noch zu starken Abweichungen der angebotenen Tests kommen kann (teilweise bis zu 18\%).

Sollte es bei 2 verschiedenen Tests zu diskordanten Ergebnissen kommen, sollte der noch verbliebene 3 . Test durchgeführt werden. Bestehen 2 diskrepante Befunde, sollte der höhere Wert bestätigt werden; dies ist mittels erneuter Testung binnen 14 Tagen möglich. Bei Werten im Grenzbereich ist eine Kontrolle in 3-6 Monaten empfohlen.

Ein Diabetes-Screening wird für Patienten mit erhöhten Fragebogen-Punktwerten (z. B. FINDRISK-Fragebogen), manifester kardiovaskulärer Erkrankung oder Vorliegen von Übergewicht und weiteren Risikofaktoren (z. B. Hypertonie, Dyslipidämie, positive Familienanamnese für Diabetes) empfohlen.

\section{Therapie}

\section{Therapie der Herzinsuffizienz bei Diabetes mellitus}

Grundsätzlich wird in den aktuellen Leitlinien der Europäischen Kardiologengesellschaft von 2016 nicht zwischen herzinsuffizienten Patienten mit und ohne Diabetes unterschieden [1]. Dies ist zum aktuellen Zeitpunkt vor allem auf die noch fehlende Evidenz von Therapieunterschieden und entsprechender Studien zurückzuführen.

\section{Therapie der Herzinsuffizienz bei Diabetes mellitus und erhaltener Pumpfunktion}

Aus der aktuellen Datenlage und den Studienergebnissen lassen sich keine speziellen Therapien der Herzinsuffizienz mit erhaltener Pumpfunktion ableiten, die zu einer Verbesserung der Prognose führen. Auch in der im letzten Jahr mit Spannung erwarteten PARAGON-HF-Studie (Angiotensin-Neprilysin Inhibition in Heart Failure with Preserved Ejection Fraction), in der die Wirkung von Sacubitril/Valsartan vs. Valsartan bei Patienten mit einer Ejektionsfraktion $\geq 45 \%$ untersucht wurde, zeigte sich kein signifikanter Unterschied im primären Endpunkt [11]. Haupttherapieziel ist es daher, die ventrikuläre Pumpfunktion und das Wohlbefinden zu erhalten und ein Fortschreiten der Erkrankung - sowie die damit einhergehende zunehmende Symptomlast und Funktionsverschlechterung - aufzuhalten bzw. zumindest zu verlangsamen. Daher sollten entsprechende Komorbiditäten, wie z. B. COPD, arterielle Hypertonie oder chronische Niereninsuffizienz, optimal eingestellt und behandelt werden. Auch asymptomatische Patienten sollen mit einem ACE-Hemmer (ACEl; ACE = AngiotensinConverting-Enzym) oder Angiotensin-Rezeptorblocker (ARB) behandelt werden. Bei kardiovaskulären Hochrisikopatienten sollte entsprechend der 2016 publizierten ESCLeitlinie zur Hyperlipidämie eine Statintherapie initiiert werden.

Therapie der Herzinsuffizienz bei Diabetes mellitus und reduzierter Pumpfunktion

Jeder symptomatische Patient mit einer NYHA-Klasse IIIV und mit einer linksventrikulären Pumpfunktion < $40 \%$ sollte eine kombinierte Basistherapie aus neurohumoraler Blockade des Angiotensin-Systems mittels ACE-Hem- 
mer (o. Sartan = ARB) und Betablocker erhalten. Hierbei sollten beide Medikamente insbesondere im ambulanten Bereich langsam bis zur maximalen (verträglichen) Dosis titriert werden.

Im Fall einer weiterhin bestehenden Symptomatik (NYHA II-IV) oder reduzierten LV-Funktion $(\leq 35 \%)$ sollte die bestehende Basistherapie um einen MineralokortikoidRezeptorantagonisten (MRA), wie Spironolacton oder Eplerenone, erweitert werden. Auch dabei wird eine langsame Dosissteigerung unter Überwachung der Retentions- und Elektrolytparameter (insbesondere des Kaliums) empfohlen.

Sollte sich der Patient unter dieser Triple-Therapie weiterhin symptomatisch zeigen oder seine LV-Funktion sich nicht über $35 \%$ erholt haben, kann bei guter Verträglichkeit der ACEI/ARB-Therapie das entsprechende Präparat gegen den Angiotensin-Neprilysin-Inhibitor (ARNI), ein Makromolekül aus Sacubitril und Valsartan (Entresto), ausgetauscht werden. Wieder gilt hier die langsame Dosissteigerung, beispielsweise mit einer Verdopplung der Dosis innerhalb von 2-4 Wochen, bis hin zur maximal verträglichen Dosis.

Falls sich der Patient zusätzlich in einem Sinusrhythmus mit einer Herzfrequenz über 70/min befinden sollte, kann der Einsatz des $\mathrm{I}_{\mathrm{f}}$-Kanalblockers Ivabradin erwogen werden. Die Zielherzfrequenz bei Herzinsuffizienz liegt bei unter 70 Schlägen pro Minute. Zeigt sich im Sinusrhythmus elektrokardiografisch eine QRS-Dauer $\geq 130 \mathrm{~ms}$ mit Linksschenkelblockmorphologie, kann die Implantation eines CRT-Implantats (CRT = „cardiac resynchronisation therapy“) erwogen werden.

Zusätzlich sollten bereits bei ersten Zeichen der Kongestion zu jeder Zeit Diuretika verordnet werden. Bei klinischer Stabilität kann deren Dosis im Verlauf langsam reduziert werden; jedoch sollte auf ein komplettes Absetzen verzichtet werden, um das Risiko einer hydropischen Dekompensation nicht zu erhöhen.

Sollte es unter den zuvor aufgeführten Therapiemaßnahmen nicht zu einer Symptombesserung des Patienten kommen, kann eine Therapie mit Digitalis-Präparaten zur weiteren Kontrolle der Herzfrequenz, sowohl bei Sinusrhythmus als auch bei Vorhofflimmern, erwogen werden. Der Einsatz von Digitalis bei Herzinsuffizienz (HFrEF) und Vorhofflimmern wird jedoch kontrovers diskutiert, da Daten eine höhere Mortalität unter dieser Therapie nahelegen. Dies zeigte sich jedoch in einer weiteren Metaanalyse nicht.

Eine ICD-Implantation ist nach Leitlinien für Patienten mit ischämischer oder dilatativer Kardiomyopathie vorgesehen, die zuvor mindestens 3 Monate eine optimale medikamentöse Therapie erhalten haben, eine Lebenserwar- tung mit gutem funktionellem Status von mehr als 12 Monaten aufweisen und deren LVEF $\leq 35 \%$ liegt.

\section{Therapie des Diabetes bei Herzinsuffizienz}

Lange galt, dass sich die Therapie des Diabetes bei kardialen Vorerkrankungen und bei Herzinsuffizienz im Speziellen nicht wesentlich von der Standardtherapie des Diabetes unterscheidet. Im Hinblick auf die aktuelle Datenlage ist dies jedoch nicht mehr gültig. Insbesondere die gute Datenlage der SGLT2-Inhibitoren hat diese Substanzklasse bei Patienten mit existenter Herzinsuffizienz in den Fokus gerückt. Dies spiegelt sich sowohl im 2019er-Update des Konsensus-Papiers der Europäischen und Amerikanischen Diabetesgesellschaft (EASD und ADA) als auch in der Leitlinie zum Diabetes/Prädiabetes und kardiovaskuläre Erkrankungen der Europäischen Kardiologengesellschaft (ESC) mit einem bevorzugten Einsatz von SGLT2Inhibitoren bei Patienten mit Diabetes und Herzinsuffizienz wider [12, 13]. Zusätzlich gilt es in Bezug auf die restlichen Substanzklassen weitere Besonderheiten mit Blick auf die Herzinsuffizienz zu beachten.

Im Folgenden wird zunächst die Datenlage zur Therapie mit SGLT2-Inhibitoren dargestellt. Anschließend werden die weiteren möglichen Therapieoptionen in alphabetischer Reihenfolge besprochen. Die möglichen Wirkungen der verschiedenen Antidiabetika auf die Herzinsuffizienz werden in $>$ Tab. 1 zusammengefasst.

\section{SGLT2-Inhibitoren}

Die Hemmung von SGLT2-Proteinen ( $S G L T=$, sodium dependent glucose transporter") im proximalen Tubulus der Niere führt zu einer Glukosurie und hat mehrere günstige Effekte: Zum einen werden $80-100 \mathrm{~g}$ Glukose pro Tag ausgeschieden, was etwa $400 \mathrm{kcal}$ entspricht. Zum anderen wird die Natriurese gesteigert, was sich hämodynamisch auswirkt, da der Blutdruck sinkt und der linksventrikuläre Massenindex abnimmt. Die Patienten verlieren bis zu $4 \mathrm{~kg}$ an Gewicht (aktuelle Übersicht der Effekte einer SGLT-Inhibition in [14]).

Die kardiale Sicherheit der verschiedenen Substanzen wurde in großen klinischen Studien untersucht. Hierbei zeigten sich deutlich positive kardiovaskuläre Effekte, die den bevorzugten Einsatz der Substanzklasse begründen:

- EMPA-REG OUTCOME (Empagliflozin Cardiovascular

Outcome Event Trial in Type 2 Diabetes Mellitus

Patients) war die erste Studie, die unter Therapie mit Empagliflozin günstige Effekte hinsichtlich eines kombinierten kardiovaskulären Endpunkts aus kardiovaskulärem Tod, nicht tödlichem Schlaganfall und Herzinfarkt zeigte. Ferner war die Gesamtmortalität hochsignifikant vermindert. Die Analyse sekundärer Endpunkte zeigte zusätzlich eine hochsignifikante Abnahme der Hospitalisierungsraten für Herzinsuffi- 


\begin{tabular}{|l|l|}
\hline Substanz & Einfluss auf die Herzinsuffizienz \\
\hline Thiazolidindione/Glitazone & ungünstig \\
\hline DPP-4-Hemmer & Saxagliptin: ungünstig \\
\hline & $\begin{array}{l}\text { Sitagliptin, Alogliptin, Linagliptin: neutral } \\
\text { Lixisenatide, Liraglutide, Semaglutide, Exenatide: neutral }\end{array}$ \\
\hline GLP-1-Rezeptoragonisten & widersprüchliche Datenlage \\
\hline Insulin & möglicherweise ungünstig \\
\hline Sulfonylharnstoffe & Acarbose: neutral \\
\hline Alpha-Glukosidase-Hemmer & möglicherweise günstig \\
\hline Metformin & Empagliflozin, Canagliflozin, Dapagliflozin: günstig \\
\hline SGLT2-Inhibitoren & \\
\hline
\end{tabular}

rot = ungünstig; gelb = neutral oder widersprüchliche Datenlage; grün = günstig; die zugrunde liegende Referenzliste kann bei den Autoren erfragt werden

- Tab. 1 Wirkung der verschiedenen antidiabetischen Medikamente auf die Herzinsuffizienz.

zienz um $35 \%$; dieses Ergebnis war unabhängig vom Vorbestehen einer Herzinsuffizienz [15].

- Ähnliche Daten gibt es für Canagliflozin anhand des Integrated CANVAS Program (CANagliflozin cardioVascular Assessment Study \& A Study of the Effects of Canagliflozin [JNJ-28 431 754] on Renal Endpoints in Adult Participants With Type 2 Diabetes Mellitus). Auch hier waren die Hospitalisierungsraten um $33 \%$ niedriger und der primäre kombinierte Endpunkt aus kardiovaskulärem Tod, nicht tödlichem Schlaganfall und Herzinfarkt um $14 \%$ vermindert. Jedoch zeigte sich keine signifikante Verringerung der Gesamt- oder kardiovaskulären Mortalität unter Canagliflozin [16].

- In ähnlicher Weise waren die kardiovaskuläre Mortalität und eine Hospitalisierung wegen Herzinsuffizienz in der DECLARE-TIMI58-Studie (Multicenter Trial to Evaluate the Effect of Dapagliflozin on the Incidence of Cardiovascular Events) bei Patienten mit Diabetes und kardiovaskulärem Risikoprofil um $17 \%$ vermindert [17].

Auf dem Boden dieser Daten wurden verschiedene Studien initiiert, die den Effekt der verschiedenen SGLT2-Inhibitoren auf die Herzinsuffizienz auch unabhängig von einem Diabetes untersuchten. Die erste Studie, die im letzten Jahr diesbezüglich veröffentlicht wurde, ist die DAPA-HF-Studie (Dapagliflozin in Patients with Heart Failure and Reduced Ejection Fraction) [18]. In dieser Studie wurden 4744 Patienten mit HFrEF (linksventrikuläre Ejektionsfraktion $\leq 40 \%$ ) auf Dapagliflozin (10 mg 1-mal täglich) oder Placebo zusätzlich zur Standard-Herzinsuffizienzbehandlung eingestellt. Nach einem medianen Follow-up von 18,2 Monaten verringerte sich das relative Risiko für Herzinsuffizienz assoziierte Ereignisse und kardialen Tod signifikant um $26 \%$ in der mit Dapagliflozin behandelten Gruppe. Auch die Gesamtmortalität konnte relativ um $17 \%$ gesenkt werden. In der Studie hatte $45 \%$ der Probanden einen Typ-2-Diabetes, $55 \%$ hatten keinen Diabetes. In einer prädefinierten Subgruppe zeigte sich, dass der Benefit der Substanz unabhängig von einem vorliegenden Diabetes mellitus war. Aktuell ist die Substanz jedoch nur zur Behandlung des Typ-2-Diabetes mit einer eGFR $\geq 60 \mathrm{ml} / \mathrm{min}$ zugelassen und sollte in diesem Patientenkollektiv bevorzugt zur Therapie der Herzinsuffizienz eingesetzt werden.

\section{Alpha-Glukosidase-Hemmer}

Alpha-Glukosidase-Hemmer verhindern die intestinale Glukoseaufnahme im Dünndarm und verhelfen somit zu einer Normalisierung des Blutzuckers. Häufig führen sie jedoch zu gastrointestinalen Nebenwirkungen, wie z. B. Diarrhö oder Flatulenz, was die Therapieadhärenz der darunter leidenden Patienten beeinflusst.

In Bezug auf die Herzinsuffizienz gibt es keine größer angelegten Studien. Die Wirkung wird gegenwärtig als neutral angesehen ( $\triangleright$ Tab. 1).

\section{DPP-4-Hemmer}

Dipeptidyl-Peptidase-4-Hemmer (DPP-4-Hemmer) verlängern die Halbwertszeit der Inkretinhormone Glucagonelike Peptide 1 (GLP-1) und Gastric inhibitory Polypeptide (GIP), die ihrerseits glukoseabhängig die Insulinsekretion erhöhen und die Glukagonausschüttung vermindern. In Monotherapie kommt es selten zu einer Hypoglykämie; ergänzt man jedoch eine bestehende SulfonylharnstoffTherapie um DPP-4-Hemmer, so kommt es zu einem um 50 \% erhöhten Hypoglykämie-Risiko.

DPP-4-Hemmer sind in der klinischen Anwendung bei Patienten mit Herzinsuffizienz sicher -mit Ausnahme von Saxagliptin:

- Saxagliptin führte in der SAVOR-TIMI53-Studie (SAVOR = Saxagliptin and Cardiovascular Outcomes in Patients with Type 2 Diabetes Mellitus) zu einer vermehrten Hospitalisierungsrate aufgrund von Herzin- 
suffizienz; hierbei handelt es sich jedoch nicht um einen Klasseneffekt.

- Für Sitagliptin zeigte sich in der in TECOS-Studie (TECOS = Trial Evaluating Cardiovascular Outcomes with Sitagliptin) und für Linagliptin in der CARMELINAStudie (CARMELINA = Cardiovascular and Renal Microvascular Outcome Study With Linagliptin in Patients With Type 2 Diabetes Mellitus) überhaupt keine Risikoerhöhung für die Hospitalisierung wegen Herzinsuffizienz.

- Für Alogliptin fand sich in EXAMINE (Examination of Cardiovascular Outcomes With Alogliptin Versus Standard of Care in patients with type 2 diabetes mellitus and acute coronary syndrome) im Trend eine nicht signifikante Risikoerhöhung.

Alle Studien mit DDP-4-Inhibitoren demonstrierten kardiovaskuläre Sicherheit, eine Überlegenheit bezüglich kardiovaskulärer Endpunkte konnte bisher nicht nachgewiesen werden.

\section{Glitazone (Thiazolidindione)}

Glitazone erhöhen die Insulinsensitivität, doch verursachen sie eine Natrium- und damit einhergehende Wasserretention. Daraus ergibt sich ein erhöhtes Risiko für eine hydropische kardiale Dekompensation bei herzinsuffizienten Patienten.

\section{Merke}

Die Anwendung von Glitazonen bei Patienten mit Herzinsuffizienz ist daher kontraindiziert [1].

\section{GLP-1-Rezeptoragonisten}

GLP-1-Rezeptoragonisten werden mittels subkutaner Injektion zugeführt. Normalerweise wird GLP-1 nahrungsabhängig im Dünndarm produziert, gemeinsam mit dem Inkretinhormon GIP. Beide Hormone bewirken eine glukoseabhängige Insulinsekretion und regulieren die Glukagonsekretion herab. Dies führt u. a. zu einer Gewichtsabnahme, Senkung des Blutdrucks und einer günstigen Veränderung des Lipidprofils:

- Liraglutid und Semaglutid zeigten eine signifikante Verbesserung des kombinierten kardiovaskulären Endpunkts aus kardiovaskulärer Mortalität, nicht tödlichem Myokardinfarkt und nicht tödlichem Schlaganfall gegenüber einer Placebo-Therapie [19, 20].

- In gleicher Weise konnte dies für Albiglutide in der HARMONY-Studie (HARMONY = Effect of Albiglutide, When Added to Standard Blood Glucose Lowering Therapies, on Major Cardiovascular Events in Subjects With Type 2 Diabetes Mellitus) [21] und für Dulaglutid in der REWIND-Studie (Dulaglutide and cardiovascular outcomes in type 2 diabetes) [22] gezeigt werden.

Die kardiovaskulär günstigen Effekte dieser Substanzen sind am ehesten dadurch zu erklären, dass die Arterio- sklerose-assoziierten Endpunkte vermindert werden. In Bezug auf die Hospitalisierung wegen Herzinsuffizienz waren diese Substanzen in den genannten Studien neutral.

\section{Merke}

GLP-1-Agonisten mit nachgewiesenem kardialem Nutzen gelten gemeinsam mit SGLT2-Inhibitoren als Mittel der ersten Wahl bei kardiovaskulär erkrankten Patienten mit Diabetes mellitus [13].

\section{Insulin}

Die subkutane Insulintherapie stellt meist die Endstrecke der Therapie des Typ-2-Diabetes dar und ist indiziert, wenn andere Therapien nicht ausreichen, um eine glykämische Kontrolle zu erreichen.

Die ORIGIN-Studie (ORIGIN = Outcome Reduction With Initial Glargine Intervention) zeigte im Jahr 2012, dass ein leichter, nicht signifikanter Trend hin zur Verminderung der Hospitalisierungen aufgrund einer Herzinsuffizienz unter Therapie mit dem langwirksamen Insulin glargin bestand (Hazard Ratio 0,95; 4,9\% vs. 5,5\%; $p=0,16$ ). Basierend auf diesen Ergebnissen der insgesamt 12537 eingeschlossenen Patienten kann von der kardiovaskulären Sicherheit einer Insulintherapie im Rahmen der Herzinsuffizienz ausgegangen werden.

\section{Metformin}

Metformin ist auch nach dem aktuellen, 2019 veröffentlichten gemeinsamen Konsensus-Beschluss von ADA und EASD weiterhin die Basistherapie des Diabetes mellitus bei kardial vorerkrankten Patienten, wohingegen die kardiologische Leitlinie Metformin nicht mehr als Erstlinientherapie sieht [13]. Aufgrund der aktuellen Zulassungssituation ist jedoch eine Gabe von Metformin, soweit verträglich, erforderlich. In der Vergangenheit war unklar, ob es unter Metformin im Rahmen einer Herzinsuffizienz und chronischen Niereninsuffizienz zu vermehrten Laktatazidosen kommen könnte. Neuere Studien demonstrieren jedoch einen Benefit der Substanz: So zeigten Evans und Kollegen, dass die Mortalität unter Metformin-Therapie sogar signifikant abnahm (HR 0,76). Ebenfalls zeigte sich ein nicht signifikanter Trend zugunsten einer verminderten Hospitalisierungsrate (HR 0,93) [23].

Neben der Senkung der Blutglukose verringert eine Metformin-Therapie das Körpergewicht und steigert die Lipolyse mit konsekutiver Absenkung der LDL-Cholesterinund Triglyzeridspiegel. Gemäß aktuellen Empfehlungen und der Fachinformation kann eine Metformin-Therapie auch unter einer GFR $<45 \mathrm{ml} /$ min fortgeführt werden wobei es wichtig ist, auf eine adäquate Dosisverringerung zu achten. Ferner sollte die Nierenfunktion regelmäßig kontrolliert werden, um bei Verschlechterung rechtzeitig 
mit dem Absetzen des Medikaments reagieren zu können.

\section{Merke}

Zusammenfassend ist Metformin als sichere Substanz zur Anwendung im Rahmen einer antidiabetischen Therapie bei herzinsuffizienten Patienten zugelassen und als Mittel der ersten Wahl empfohlen.

Zum aktuellen Zeitpunkt fehlen jedoch prospektive Endpunktstudien zur positiven Auswirkung der MetforminTherapie auf eine bestehende Herzinsuffizienz.

\section{Sulfonylharnstoffe}

Sulfonylharnstoffe bewirken eine vermehrte pankreatische Insulinausschüttung sowie eine Hemmung der hepatischen Glukoneogenese. Da die Insulinsekretion durch Sulfonylharnstoffe glukoseunabhängig verläuft, treten häufiger Hypoglykämien auf. Dieser Effekt ist bei Sulfonylharnstoffen der neuen Generationen geringer.

Abgesehen von einer Studie mit 10089 gematchten Patienten, die zusätzlich zu einer Basistherapie mit Metformin entweder Sulfonylharnstoffe oder DPP-4-Hemmer erhielten, gibt es keine Daten, die einen kardiovaskulären Vorteil dieser Substanzgruppe belegen [24]. Diese Studie zeigte einen Vorteil von DPP-4-Hemmern gegenüber der Therapie mit Sulfonylharnstoffen. Die kardiovaskuläre Sicherheit scheint nach gegenwärtigem Kenntnisstand jedoch gegeben, sodass Sulfonylharnstoffe bei Herzinsuffizienz verordnet werden können.

\section{FALLBEISPIEL}

Ein 58-jähriger Patient mit koronarer 3-Gefäßerkrankung und mittelgradig eingeschränkter linksventrikulärer Funktion (LVEF 33 \%) bei Z. n. Vorderwandinfarkt vor 2 Jahren wird aktuell behandelt mit Azetylsalizylsäure $100 \mathrm{mg} / \mathrm{d}$, Bisoprolol $2,5 \mathrm{mg} / \mathrm{d}$ sowie Ramipril 2-mal $5 \mathrm{mg} / \mathrm{d}$, Torasemid $2,5 \mathrm{mg} / \mathrm{d}$, Eplerenon $25 \mathrm{mg} / \mathrm{d}$ und Atorvastatin $60 \mathrm{mg} / \mathrm{d}$. Der BMI beträgt $34 \mathrm{~kg} / \mathrm{m}^{2}$.

Zusätzlich hat der Patient primärphrophylaktisch einen ICD implantiert bekommen. Als Komorbidität ist seit 5 Jahren ein Diabetes mellitus Typ 2 bekannt, der bislang diätetisch versorgt wurde.

Der Patient stellt sich mit zunehmender Dyspnoe bei Belastung vor. In den Laboruntersuchungen zeigt sich ein NT-proBNP von 3521 pg/ml, eine eingeschränkte Nierenfunktion mit einer GFR von $46 \mathrm{ml} / \mathrm{min}$ pro Kilogramm Körpergewicht sowie ein $\mathrm{HbA}_{1 \mathrm{c}}$-Wert von $7,8 \%$.
Nach Intensivierung der Diuretika-Therapie und Umsetzen von Ramipril auf Sacubitril/Valsartan gehen die Beschwerden des Patienten deutlich zurück. Bezüglich des Diabetes mellitus lehnt der Patient eine Injektionstherapie ab, sodass eine orale antidiabetische Therapie mit Metformin 1-mal 500 mg (verringerte Dosis bei eingeschränkter Nierenfunktion) und Semaglutid $(0,25 \mathrm{mg} 1$-mal pro Woche, Steigerung nach 4 Wochen auf $0,5 \mathrm{mg} 1$-mal pro Woche) begonnen wird. Ihm wird empfohlen, den $\mathrm{HbA}_{1 c^{-}}$ Wert nach 3 Monaten kontrollieren zu lassen.

Rationale hinter der Therapieentscheidung: Bei symptomatischen Patienten mit einer Ejektionsfraktion unter $35 \%$ sollte der ACE-Hemmer auf Sacubitril/Valsartan umgesetzt werden. Zusätzlich wird die diuretische Therapie bei erhöhtem NT-proBNP und Stauungssymptomen temporär intensiviert. Für eine effektivere antidiabetische Therapie käme eigentlich - aufgrund des gezeigten kardiovaskulären Benefits - ein SGLT2-Inhibitor infrage. Dieser scheidet jedoch bei einer GFR von 46 aus. Aufgrund der Adipositas und dem kardiovaskulären Risikoprofil wurde eine Therapie mit Semaglutid gewählt, die zu einer deutlichen Gewichtsreduktion führt.

\section{Fazit}

Der Diabetes mellitus ist eine sehr wichtige Komorbidität bei Patienten mit Herzinsuffizienz, da das gemeinsame Vorliegen beider Erkrankungen die Prognose der Patienten deutlich verschlechtert. Es ist daher essenziell, beide Erkrankungen frühzeitig zu diagnostizieren und leitliniengerecht zu behandeln.

Die Therapie der Herzinsuffizienz unterscheidet sich bei Patienten mit Diabetes mellitus nicht von denen ohne Diabetes. Dapagliflozin hat im letzten Jahr einen hochsignifikanten Effekt sowohl auf Herzinsuffizienz assoziierte Ereignisse als auch auf die kardiovaskuläre und die Gesamtmortalität gezeigt. Bis zur Zulassung für die Herzinsuffizienz sollten alle Patienten mit Typ-2-Diabetes, die die aktuelle Zulassung (insbesondere die GFR-Kriterien) erfüllen, einen SGLT2-Inhibitor erhalten. Alternativ sollte ein GLP-1-Rezeptoragonist mit nachgewiesenem kardialem Benefit gewählt werden, weil es in der Therapie des Diabetes wichtig ist, Substanzen mit positivem Effekt auf das kardiovaskuläre Outcome der Patienten einzusetzen. Substanzen, die ungünstige Effekte auf die Herzinsuffizienz gezeigt haben, sollten gemieden werden. 


\section{KERNAUSSAGEN}

- Patienten mit Diabetes mellitus und Herzinsuffizienz haben ein deutlich erhöhtes kardiovaskuläres Risiko und versterben früher.

- Eine frühe Diagnostik des Diabetes und der Herzinsuffizienz ist elementar für eine gezielte Therapieeinleitung und Prognoseverbesserung.

- Die Therapie der Herzinsuffizienz unterscheidet sich nicht bei Patienten mit und ohne Diabetes.

- Bei Patienten mit Herzinsuffizienz und Typ-2-Diabetes und einer GFR $\geq 60 \mathrm{ml} / \mathrm{min} / 1,73 \mathrm{~m}^{2}$ sollte primär ein SGLT2-Inhibitor eingesetzt werden.

- Eine strenge Einstellung der Risikofaktoren nach aktuellen Leitlinien verringert die kardiovaskuläre Mortalität und Hospitalisierungsrate.

- Glitazone und Saxagliptin gehen mit einer erhöhten Hospitalisierungsrate einher und sollten bei Herzinsuffizienz gemieden werden.

\section{Wissenschaftlich Verantwortlicher gemäß Zertifizierungsbestimmungen}

Wissenschaftlich Verantwortlicher gemäß Zertifizierungsbestimmungen für diesen Beitrag ist Priv.-Doz. Dr. med. Katharina A. Schütt, Aachen.

\section{Interessenkonflikt}

\section{Erklärung zu finanziellen Interessen}

Forschungsförderung erhalten: ja, von einer anderen Institution (Pharma- oder Medizintechnikfirma usw.); Honorar/geldwerten Vorteil für Referententätigkeit erhalten: ja, von einer anderen Institution (Pharma- oder Medizintechnikfirma usw.); Bezahlter Berater/interner Schulungsreferent/Gehaltsempfänger: ja, von einer anderen Institution (Pharma- oder Medizintechnikfirma usw.); Patent/Geschäftsanteile/Aktien (Autor/ Partner, Ehepartner, Kinder) an Firma (Sponsor der Veranstaltung): nein; Patent/Geschäftsanteile/Aktien (Autor/Partner, Ehepartner, Kinder) an Firma (Nicht-Sponsor der Veranstaltung): nein.

Erklärung zu nichtfinanziellen Interessen

Katharina A. Schütt: Deutsche Gesellschaft für Kardiologie (Stellvertretender Sprecher der AG Herz und Diabetes) Nukleus Mitglied der Deutschen Arteriosklerose Gesellschaft.

Autorinnen/Autoren

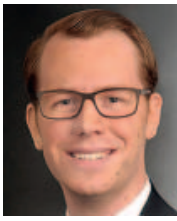

\section{Philipp H. Baldia}

Dr. med., seit 2018 Assistenzarzt in der Med. Klinik I am Universitätsklinikum RWTH Aachen. Seit 2015 Forschung (Arbeitsgruppe Dr. K. Schütt) über kardiovaskuläre Hochrisikopatienten mit Diabetes mellitus und/oder chronischer Nierenerkrankung.

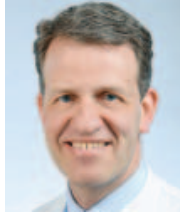

\section{Nikolaus Marx}

Prof. Dr. med., Medizinstudium in Mainz, Genf und Düsseldorf. 1994 Approbation und Promotion. 1994-1997 Assistenzarzt an der 1. Medizinischen Klinik und Poliklinik der TU München, am Klinikum rechts der Isar und Deutschen Herzzentrum. 1997-1999 wissenschaftliche Tätigkeit an der Harvard Medical School, Boston, USA. 19992009 Assistenzarzt, Oberarzt und Leitender Oberarzt der Abteilung Innere Medizin II am Universitätsklinikum Ulm. Seit November 2009 Direktor der Medizinischen Klinik I am Universitätsklinikum Aachen.

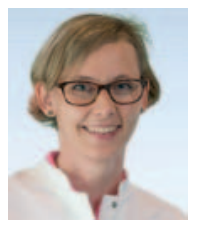

\section{Katharina A. Schütt}

Priv.-Doz. Dr. med., Fachärztin für Innere Medizin und Kardiologie. Seit 2016 kardiologische Oberärztin am Universitätsklinikum RWTH Aachen, Leiterin der Herzinsuffizienz-Ambulanz. Schwerpunkte: kardiovaskuläre Hochrisikopatienten mit Diabetes mellitus und/oder chronischer Nierenerkrankung.

\section{Korrespondenzadresse}

Priv.-Doz. Dr. med. Katharina Schütt

Universitätsklinikum RWTH Aachen

Medizinische Klinik I - Kardiologie, Angiologie und

Internistische Intensivmedizin

Pauwelsstraße 30

52074 Aachen

kschuett@ukaachen.de

\section{Zitierweise für diesen Artikel}

Dtsch Med Wochenschr 2020; 145: Seite 1258-Seite 1270, 10.1055/a-1117-8446

Dieser Beitrag ist eine aktualisierte Version des Artikels: Baldia P, Marx N, Schütt K. Diabetes und Herzinsuffizienz. Kardiologie up2date 2019; 15(01): 27-39

\section{Literatur}

[1] Ponikowski P, Voors AA, Anker SD et al. 2016 ESC Guidelines for the diagnosis and treatment of acute and chronic heart failure. Eur Heart ] 2016; 37: 2129-2200. doi:10.1093/eurheartj/ ehw128

[2] Nichols GA, Gullion CM, Koro CE et al. The incidence of congestive heart failure in type 2 diabetes: an update. Diabetes Care 2004; 27: 1879-1884. doi:10.2337/diacare.27.8.1879

[3] Bahrami H, Bluemke DA, Kronmal R et al. Novel Metabolic Risk Factors for Incident Heart Failure and Their Relationship With Obesity. The MESA (Multi-Ethnic Study of Atherosclerosis) Study. J Am Coll Cardiol 2008; 51: 1775-1783. doi:10.1016/ j.jacc.2007.12.048

[4] Nichols GA, Koro CE, Gullion CM et al. The incidence of congestive heart failure associated with antidiabetic therapies. Diabetes Metab Res Rev 2005; 21: 51-57. doi:10.1002/dmrr.480

[5] Van Melle JP, Bot M, De Jonge P et al. Diabetes, glycemic control, and new-onset heart failure in patients with stable coronary artery disease: Data from the heart and soul study. Diabetes Care 2010; 33: 2084-2089. doi: 10.2337/dc10-0286

[6] Cavender MA, Steg PG, Smith SC et al. Impact of Diabetes Mellitus on Hospitalization for Heart Failure, Cardiovascular Events, 
and Death: Outcomes at 4 Years from the Reduction of Atherothrombosis for Continued Health (REACH) Registry. Circulation 2015; 132: 923-931. doi:10.1161/CIRCULATIONAHA. 114.014796

[7] Matsue Y, Suzuki M, Nakamura R et al. Prevalence and Prognostic Implications of Pre-Diabetic State in Patients With Heart Failure. Circ J 2011; 75: 2833-2839. doi:10.1253/circj.CJ-110754

[8] Lehrke M, Marx N. Diabetes Mellitus and Heart Failure. Am J Cardiol 2017; 120: S37-S47. doi:10.1016/j.amjcard. 2017.05.014

[9] Kristensen SL, Preiss D, Jhund PS et al. Risk Related to PreDiabetes Mellitus and Diabetes Mellitus in Heart Failure with Reduced Ejection Fraction: Insights from Prospective Comparison of ARNI with ACEI to Determine Impact on Global Mortality and Morbidity in Heart Failure Trial. Circ Hear Fail 2016; 9: 1-12. doi:10.1016/j.amjcard.2017.05.014

[10] Gustafsson I, Brendorp B, Seibaek M et al. Danish Investigatord of A, Mortality on Dofetilde Study G. Influence of diabetes and diabetes-gender interaction on the risk of death in patients hospitalized with congestive heart failure. J Am Coll Cardiol 2004; 43: 771-777. doi:10.1016/j.jacc.2003.11.024

[11] Solomon SD, McMurray JJV, Anand IS et al. AngiotensinNeprilysin Inhibition in Heart Failure with Preserved Ejection Fraction. N Engl ] Med 2019; 381: 1609-1620. doi:10.1056/ NEJMoa1908655

[12] Buse JB, Wexler DJ, Tsapas A et al. 2019 Update to: Management of Hyperglycemia in Type 2 Diabetes, 2018. A Consensus Report by the American Diabetes Association (ADA) and the European Association for the Study of Diabetes (EASD). Diabetes Care 2020; 43: 487-493. doi:10.2337/dci19-0066

[13] Cosentino F, Grant PJ, Aboyans V et al. 2019 ESC Guidelines on diabetes, pre-diabetes, and cardiovascular diseases developed in collaboration with the EASD: The Task Force for diabetes, pre-diabetes, and cardiovascular diseases of the European Society of Cardiology (ESC) and the European Association for the Study of Diabetes (EASD). Eur Heart J 2019; 41: 255-323. doi:10.1093/eurheartj/ehz486

[14] Thomas MC, Cherney DZI. The actions of SGLT2 inhibitors on metabolism, renal function and blood pressure. Diabetologia 2018; 61: 2098-2107. doi:10.1007/s00125-018-4669-0

[15] Zinman B, Wanner C, Lachin JM et al. Empagliflozin, Cardiovascular Outcomes, and Mortality in Type 2 Diabetes. N Engl J Med 2015; 373: 2117-2128. doi:10.1056/NEJMoa1504720
[16] Neal B, Perkovic V, Mahaffey KW et al. Canagliflozin and Cardiovascular and Renal Events in Type 2 Diabetes. N Engl J Med 2017; 377: 644-657. doi:10.1056/NEJMoa1611925

[17] Wiviott SD, Raz I, Bonaca MP et al. Dapagliflozin and Cardiovascular Outcomes in Type 2 Diabetes. N Engl ] Med 2019; 380: 347-357. doi:10.1056/NEJMoa1812389

[18] McMurray JJV, Solomon SD, Inzucchi SE et al. Dapagliflozin in Patients with Heart Failure and Reduced Ejection Fraction. N Engl J Med 2019; 381: 1995-2008. doi:10.1056/ NEJMoa1911303

[19] Marso SP, Daniels GH, Brown-Frandsen K et al. Liraglutide and Cardiovascular Outcomes in Type 2 Diabetes. N Engl ] Med 2016; 375: 311-322. doi:10.1056/NEJMoa1603827

[20] Marso SP, Bain SC, Consoli A et al. Semaglutide and Cardiovascular Outcomes in Patients with Type 2 Diabetes. N Engl J Med 2016; 375: 1834-1844. doi:10.1056/NEJMoa1607141

[21] Hernandez AF, Green JB, Janmohamed S et al. Albiglutide and cardiovascular outcomes in patients with type 2 diabetes and cardiovascular disease (Harmony Outcomes): a double-blind, randomised placebo-controlled trial. Lancet 2018; 392: 15191529. doi:10.1016/s0140-6736(18)32261-x

[22] Gerstein HC, Colhoun HM, Dagenais GR et al. Dulaglutide and cardiovascular outcomes in type 2 diabetes (REWIND): a double-blind, randomised placebo-controlled trial. Lancet 2019; 394: 121-130. doi:10.1016/s0140-6736(19)31149-3

[23] Aguilar D, Chan W, Bozkurt B et al. Metformin use and mortality in ambulatory patients with diabetes and heart failure. Circ Heart Fail 2011; 4: 53-58. doi:10.1161/circheartfailure.110.952556

[24] Ou SM, Shih C], Chao PW et al. Effects on Clinical Outcomes of Adding Dipeptidyl Peptidase-4 Inhibitors Versus Sulfonylureas to Metformin Therapy in Patients With Type 2 Diabetes Mellitus. Ann Intern Med 2015; 163: 663-672. doi:10.7326/m15-0308

Bibliografie

DOI https://doi.org/10.1055/a-1117-8446

Dtsch Med Wochenschr 2020; 145: 1258-1270

(C) Georg Thieme Verlag KG, Stuttgart · New York ISSN 0012-0472 


\section{Punkte sammeln auf CME.thieme.de}

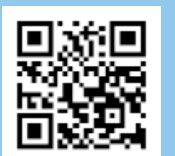

Diese Fortbildungseinheit ist in der Regel 12 Monate online für die Teilnahme verfügbar.

Den genauen Einsendeschluss finden Sie unter https://eref.thieme.de/CXEMFYX.

Sollten Sie Fragen zur Online-Teilnahme haben, finden Sie unter https://cme.thieme.de/hilfe

eine ausführliche Anleitung. Wir wünschen viel Erfolg beim Beantworten

der Fragen!

Unter https://eref.thieme.de/CXEMFYX oder über den QR-Code kommen Sie direkt zur Startseite des Wissenstests.

VNR 2760512020158723581

\section{Frage 1}

Welche der folgenden Aussagen ist nicht richtig?

A Die Herzinsuffizienz tritt bei Individuen mit Diabetes genauso häufig und in ähnlichem Alter auf.

B Mit zunehmendem Lebensalter steigt auch die Inzidenz der Herzinsuffizienz an.

C Bereits ein „Prädiabetes“ hat Auswirkungen hinsichtlich des Auftretens einer Herzinsuffizienz.

D Es wird ein weiterer Anstieg der Prävalenz des Diabetes mellitus erwartet.

E Die Kombination aus Diabetes mellitus und Herzinsuffizienz ist mit einer schlechten Prognose vergesellschaftet.

\section{Frage 2}

Welche der folgenden Aussagen zur Häufigkeit von Diabetes mellitus und Herzinsuffizienz ist richtig?

A Bis zum Jahr 2040 werden weltweit etwa 1 Milliarde Menschen von einem Diabetes mellitus betroffen sein.

B In Europa sind aktuell etwa 30 Millionen Menschen vom Diabetes mellitus betroffen.

C Aktuell sind weltweit knapp 500 Millionen Menschen im Alter über 20 Jahren von einem Diabetes mellitus betroffen.

D Die Prävalenz der Herzinsuffizienz nimmt bei den über 70 Jahre alten Menschen auf über $20 \%$ zu.

E Daten aus verschiedenen Registerstudien legen nahe, dass die Prävalenz des Diabetes bei Herzinsuffizienz bei über $50 \%$ liegt.

\section{Frage 3}

Welche der folgenden Aussagen zur Diagnostik der Herzinsuffizienz ist richtig?

A Zum Nachweis der Herzinsuffizienz sollte breit und symptomunabhängig echokardiografisch gescreent werden.

B Anamnese und Klinik spielen bei der Diagnostik eine nur untergeordnete Rolle.

C Bei klinischem Verdacht auf eine Herzinsuffizienz sollten die natriuretischen Peptide bestimmt werden.

D Für Patienten mit Diabetes mellitus unterscheidet sich der Diagnosealgorithmus wesentlich von Patienten ohne Diabetes mellitus.

E Orthopnoe ist kein typisches Symptom der Herzinsuffizienz. Eine weitere ätiologische Abklärung ist nicht zwingend erforderlich.

\section{Frage 4}

Welches der folgenden Kriterien wird gemäß ESC bei der Diagnostik einer HFpEF nicht angewendet?

A Symptome der Herzinsuffizienz

B erhöhte Spiegel natriuretischer Peptide

C nachgewiesenes Vorhofflimmern

D $\quad$ LVEF $\geq 50 \%$

E Nachweis struktureller oder funktioneller Veränderungen

\section{Frage 5}

Welche der folgenden Aussagen zur Diagnostik des Diabetes mellitus Typ 2 ist richtig?

A Ein 1-malig erfasster HbA1c von 6,2\% gilt als beweisend für einen manifesten Diabetes mellitus.

B Eine Gelegenheitsplasmaglukose über $200 \mathrm{mg} / \mathrm{dl}$ ist beweisend für einen Diabetes mellitus Typ 2.

C Mittel der Wahl zur Diagnostik des Diabetes mellitus Typ 2 ist der $\mathrm{HbA}_{1 \mathrm{c}}$.

D Im oralen Glukosetoleranztest wird der Plasmaglukosespiegel zum Zeitpunkt 0 und nach 3 Stunden bestimmt.

E Die Prävalenz des Diabetes bei Patienten mit Herzinsuffizienz ist so niedrig, dass ein Screening nicht zielführend ist.

- Weitere Fragen auf der folgenden Seite... 


\section{Punkte sammeln auf CME.thieme.de}

\section{Frage 6}

Welche der folgenden Aussagen zur Therapie der Herzinsuffizienz ist nicht richtig?

A Die Basistherapie der Herzinsuffizienz besteht aus einem ACEHemmer und einem Betablocker.

B Die Therapieeskalation erfolgt nach linksventrikulärer Ejektionsfraktion und klinischer Symptomatik.

C Bei Vorliegen einer NYHA II, einer Ejektionsfraktion von $30 \%$ und einem Linksschenkelblock mit einem QRS-Komplex von 156 ms ist eine kardiale Resynchronisation plus Defibrillator (CRT-D-System) indiziert.

D Im Vorhofflimmern kann eine Natriumkanalblocker-Therapie mittels Ivabradin zum Verlangsamen der Herzfrequenz verwendet werden.

E Es ist wichtig, nach Möglichkeit die angegebenen Zieldosen zur Behandlung der Herzinsuffizienz zu erreichen. Dies sollte unter Kontrolle der Retentions- und Elektrolytparameter sowie des Blutdrucks geschehen.

\section{Frage 7}

Welche der folgenden Konstellation definiert keinen Prädiabetes?
A Nüchtern-Plasmaglukose $110 \mathrm{mg} / \mathrm{dl}$
B 2-Stunden-Plasmaglukose $170 \mathrm{mg} / \mathrm{dl}$
C $\mathrm{HbA}_{1 \mathrm{c}} 6,0 \%$
D 2-Stunden-Plasmaglukose $190 \mathrm{mg} / \mathrm{dl}$
E Nüchtern-Plasmaglukose $130 \mathrm{mg} / \mathrm{dl}$

\section{Frage 8}

Welche der folgenden Aussagen zur Therapie des Diabetes ist richtig?

A Lebensstilmodifikationen spielen keine Rolle mehr in der aktuellen Therapie des Diabetes mellitus.

B Sulfonylharnstoffe sollten immer in der Therapie enthalten sein.

C Unabhängig von den Komorbiditäten sollten immer DDP-4Hemmer eingesetzt werden.

D Saxagliptin weist eine erhöhte Rate an Hospitalisierungen aufgrund von Herzinsuffizienz auf und sollte daher bei Patienten mit Herzinsuffizienz nicht eingesetzt werden.

E Metformin kann auch bei schwerer Einschränkung der Nierenfunktion weiter verordnet werden.

\section{Frage 9}

Welche der folgenden Aussagen zur Therapie des Diabetes bei Herzinsuffizienz ist nicht richtig?

A Je nach Patientenalter und Komorbiditäten sollte ein Ziel$\mathrm{HbA}_{1 \mathrm{c}}<7 \%$ bzw. 6,5\% erreicht werden.

B SGLT2-Hemmer führen zu einer Gewichts- und Blutdruckreduktion.

C GLP-1-Analoga und SGLT2-Hemmer können beide bei kardiovaskulärer Erkrankung und Diabetes eingesetzt werden.

D SGLT2-Hemmer bzw. GLP-1-Analoga mit nachgewiesenem kardialem Benefit sollten zur Einstellung eines Diabetes mellitus bei kardiovaskulärer Erkrankung herangezogen werden.

E Die Therapie mit SGLT2-Inhibitoren kann bis zu einer GFR von $45 \mathrm{ml} / \mathrm{min}$ begonnen werden.

\section{Frage 10}

Welche der folgenden Aussagen zur Therapie des Diabetes bei Herzinsuffizienz ist richtig?

A SGLT2-Inhibitoren sollten als primäre Therapie im Rahmen ihrer Zulassung eingesetzt werden.

B Da die Wirkung der Antidiabetika glukoseunabhängig ist, sind Kontrollen der Blutzuckereinstellung in der heutigen Zeit obsolet.

C Unter SGLT2-Hemmer-Therapie sollte die Uringlukose zum Monitoring bestimmt werden.

D GLP-1-Analoga werden per os verabreicht.

E Insulin hat ein erhöhtes Risiko für Hospitalisierungen aufgrund von Herzinsuffizienz und sollte gemieden werden. 